\title{
Spectral determinants for twist field correlators
}

\author{
A. V. Belitsky \\ Department of Physics, Arizona State University, Tempe, Arizona 85287-1504, USA
}

(Received 4 December 2017; published 18 April 2018)

\begin{abstract}
Twist fields were introduced a few decades ago as a quantum counterpart to classical kink configurations and disorder variables in low dimensional field theories. In recent years they received a new incarnation within the framework of geometric entropy and strong coupling limit of four-dimensional scattering amplitudes. In this paper, we study their two-point correlation functions in a free massless scalar theory, namely, twist-twist and twist-antitwist correlators. In spite of the simplicity of the model in question, the properties of the latter are far from being trivial. The problem is reduced, within the formalism of the path integral, to the study of spectral determinants on surfaces with conical points, which are then computed exactly making use of the zeta function regularization. We also provide an insight into twist correlators for a massive complex scalar by means of the Lifshitz-Krein trace formula.
\end{abstract}

DOI: 10.1103/PhysRevD.97.086008

\section{INTRODUCTION}

Two-dimensional conformal field theory on a (say, hyperelliptic) Riemann surface $\mathcal{R}$ can be reformulated as a theory on a branched covering of the complex plane, where each branch point $z_{i}$ corresponds to an insertion of a conformal primary field $V\left(z_{i}\right)[1-3]$, known as the branch point twist field. The argument goes as follows [1-3]. Consider a small vicinity of the branch point $z_{j}$ of order $N$ (with a cut emanating to infinity) on the covering of the original Riemann surface with $N$ consecutive sheets enumerated by $\ell=1, \ldots, N$ and parametrized by the single-valued coordinate $\zeta$,

$$
\zeta=\sqrt[N]{z-z_{j}}
$$

When a point $z=z_{j}+\varepsilon \mathrm{e}^{i \varphi}$ is taken around $z_{i}$, i.e., from $\varphi=0$ to $\varphi=2 \pi$, the coordinate $\zeta$ moves from the $\ell$ th to $(\ell+1)$ st sheet. Let the field theory on each sheet $\ell$ be described by an action $S_{(\ell)}=S\left[X_{(\ell)}\right]$. The complex fields $X_{(\ell)}$ belonging to different sheets do not interact with each other. We identify them across the cuts $\left[z_{i}, \infty\right)$,

$$
\left.X_{(\ell)}(z)\right|_{z \in\left[z_{j}, \infty\right)}=\left.X_{(\ell+1)}(z)\right|_{z \in\left[z_{j}, \infty\right)},
$$

and obviously cyclically identify $N+1=1$. This corresponds to gluing sheets together into the original Riemann

Published by the American Physical Society under the terms of the Creative Commons Attribution 4.0 International license. Further distribution of this work must maintain attribution to the author(s) and the published article's title, journal citation, and DOI. Funded by SCOAP ${ }^{3}$. surface. The Boltzmann weight $\mathrm{e}^{-S}$ in the partition function is then given by the sum of $N$ copies of the model on a single complex plane $S=\sum_{\ell=1}^{N} S_{(\ell)}$ and enjoys $Z_{N}$ permutation symmetry $\sigma X_{(\ell)}=X_{(\ell+1)}$. The latter can be diagonalized by forming a linear combination of fields of the $N$ copies,

$$
X_{[k]}=\sum_{\ell=1}^{N} \mathrm{e}^{2 \pi i \frac{k \ell}{N}} X_{(\ell)}, \quad \sigma X_{[k]}=\mathrm{e}^{2 \pi i \frac{k}{N}} X_{[k]} .
$$

The action can be equivalently written in terms of $N$ still decoupled copies of these, i.e., $S=\sum_{k=0}^{N-1} S\left[X_{[k]}\right]$. The above equation immediately implies that the new fields $X_{[k]}$ are not single valued but acquire a phase as their argument crosses the cuts

$$
\left.X_{[k]}\left(z_{\circlearrowleft_{j}}\right)\right|_{z \in\left[z_{j}, \infty\right)}=\left.\mathrm{e}^{2 \pi i_{N}^{k}} X_{[k]}(z)\right|_{z \in\left[z_{j}, \infty\right)} .
$$

This information can be encoded into the operator product expansion of the field $X_{[k]}(z)$ with a twist field $V_{k / N}\left(z_{j}\right)$, such that

$X_{[k]}(z) V_{k / N}\left(z_{j}\right)=\left(z-z_{j}\right)^{k / N}: X_{[k]} V_{k / N}:(z)+\cdots$.

For the entire $\mathcal{R}$, the integrand in the path integral is given by the product of Boltzmann weights $\mathrm{e}^{-S}=\prod_{k=0}^{N-1} \mathrm{e}^{-S\left[X_{[k]}\right]}$ and one introduces the corresponding branch point twist field by forming the product of twist fields over all $N$ copies,

$$
V\left(z_{j}\right)=\prod_{k=1}^{N-1} V_{k / N}\left(z_{j}\right) .
$$


Here we accounted for the fact that $V_{0}=1$. Consequently, the field theory on the original Riemann surface is reformulated in terms of the $N$ decoupled copies on the complex plane but with insertions of branch point twist fields,

$$
\begin{aligned}
\int[D X]_{\mathcal{R}} \mathrm{e}^{-S_{\mathcal{R}}} & =\int \prod_{k=0}^{N-1}\left(\left[D X_{[k]}\right] \mathrm{e}^{-S\left[X_{[k]}\right]}\right) \prod_{j} V\left(z_{j}\right) \\
& \equiv\left\langle\prod_{j} V\left(z_{j}\right)\right\rangle
\end{aligned}
$$

We implicitly imply that some (or all) of the fields can be branch point antitwists $\bar{V}\left(z_{j}\right)=\prod_{k=1}^{N-1} V_{-k / N}\left(z_{j}\right)$.

The consideration that follows is done having two particular applications of the branch point twist fields in mind. The first one is the calculation of von Neumann $S_{\mathrm{vN}}$ geometric (or entanglement) entropy [4] for a one-dimensional observer residing on a single interval $A=[0, x]$ with the rest of the real line $B=\mathbb{R} \backslash A$ being unattainable,

$$
S_{\mathrm{vN}}=-\operatorname{tr}_{\mathrm{A}} \rho_{\mathrm{A}} \ln \rho_{\mathrm{A}}, \quad \rho_{\mathrm{A}}=\operatorname{tr}_{\mathrm{B}}|\psi\rangle\langle\psi| .
$$

Here $|\psi\rangle$ is a pure state of the quantum system on $\mathbb{R}$ and traces are taken over the degrees of freedom on the labeled intervals. A standard technique for computation of $S_{\mathrm{sV}}$ is the replica trick [4,5]: introducing $N$ copies of the system, computing the Renyi entropy $S_{\mathrm{R}}$ and constructing its proper analytic continuation in $N$ allows one to compute its derivative,

$$
S_{\mathrm{R}}(N)=\operatorname{tr}_{A} \rho_{A}^{N}, \quad S_{\mathrm{vN}}=-\left.\frac{d S_{\mathrm{R}}(N)}{d N}\right|_{N \rightarrow 1} .
$$

The Renyi entropy can be cast in a form of the path integral over a hyperelliptic Riemann surface, $\zeta^{N}=z(z-x)$, with a branch cut $[0, x]$. Then according to the previous discussion, it admits (up to an overall, possibly divergent, constant) the form of the correlation function of a branch point twist field at $z_{1, \mu}=(x, 0)$ and antitwist at $z_{2, \mu}=$ $(0,0)[6]$,

$$
S_{\mathrm{R}}(N)=c_{N}\langle\bar{V}(0) V(x)\rangle .
$$

The second application pertains to the recent use of the pentagon form factor program [7-9] for computation of $L$-particle scattering amplitudes $\mathcal{A}_{L}$ in four-dimensional maximally supersymmetric Yang-Mills theory. The formalism relies on a dual description of on-shell massless amplitudes in terms of expectation values of Wilson loops stretched on a null polygonal contour tracing the path formed by the particles' momenta [10-12]. Dynamical information is then encoded through the physics of excitations $\psi$ propagating on the two-dimensional world sheet ending on the four-dimensional boundary. The amplitudes admit the following schematic representation,

$$
\begin{aligned}
\mathcal{A}_{L}= & \sum_{1,2, \ldots L-5}\left\langle 0|\hat{\mathcal{P}}| \psi_{L-5}\right\rangle \mathrm{e}^{-\tau_{L-5} E_{L-5}+i \sigma_{L-5} P_{L-5}+i \varphi_{L-5} m_{L-5}} \ldots \\
& \times\left\langle\psi_{2}|\hat{\mathcal{P}}| \psi_{1}\right\rangle \mathrm{e}^{-\tau_{1} E_{1}+i \sigma_{1} P_{1}+i \varphi_{1} m_{1}}\left\langle\psi_{1}|\hat{\mathcal{P}}| 0\right\rangle
\end{aligned}
$$

as a sum over an infinite number of the so-called flux-tube excitations and their bound states with external geometrical data $\left(\tau_{j}, \sigma_{j}, \varphi_{j}\right)$ included through the phase factors parametrized by total energy $E_{j}$, momentum $P_{j}$ and (twice the) helicity $m_{j}$ of the intermediate state $\left|\psi_{j}\right\rangle$. The physics of transitions between adjacent intermediate states is determined by matrix elements of pentagon operators $\hat{\mathcal{P}}$. At strong 't Hooft coupling $g^{2}$, integrating out all heavy fermionic and gauge modes, the only propagating degrees of freedom that remain are the six nearly massless scalars. They are described by the $\mathrm{O}(6)$ nonlinear sigma model [13]. In the ultraviolet regime, due to asymptotic freedom of the theory, the sigma model coupling vanishes and one ends up with a free theory of five massless real scalars. These propagate on a background with conical singularities at the space-time positions of pentagon operators $z_{j}=\left(\sigma_{j}, \tau_{j}\right)$ on a two-dimensional plane [14]. Each cone has an excess angle of $\pi / 2$ as it corresponds to folding the pentagon on a time-space square [15]. Thus, in the vicinity of each cone, we are dealing with $\left(1+\frac{1}{4}\right)$-sheeted Riemann surface. As a consequence, the pentagon operators $\hat{\mathcal{P}}$ take on the meaning of (fractional) branch point twist fields $V$. One subtle point that one has to keep in mind is that the introduction of twist fields requires operating with complex rather than real fields so one effectively doubles the number of degrees of freedom to construct a microscopic definition of pentagon operators. This is a common trick, successfully employed in many different circumstances, e.g., in the Ising model [16]. Merely taking the square root at the end reduces this number in half. In this manner, the (square of the) scattering amplitudes as strong coupling are given by the $(L-5)$ point correlation function of branch point twist fields $[15,17,18]$,

$$
\mathcal{A}_{L}^{2} \stackrel{g^{2} \rightarrow \infty}{=}\left\langle V\left(z_{L-5}\right) \ldots V\left(z_{1}\right) V(0)\right\rangle .
$$

In what follows, we focus on the simplest $L=6$ case, i.e., the hexagon.

The focus of our current study is on two-point correlation functions in free theories of an elementary massless complex scalar field. Our subsequent presentation is organized as follows. In the next section, we recall the definition of twist fields within the formalism of the path integral. In Sec. III, we compute the twist-twist and then, in Sec. IV, twist-antitwist correlator in massless theories by reducing them to the calculation of spectral determinants regularized by means of the zeta function. While the twisttwist sector enjoys the conventional powerlike scaling with the distance typical for theories with infinite correlation 
length, the latter one acquires an additional logarithmic factor intrinsic to logarithmic conformal field theories [19]. Finally, we conclude and provide a sketch for generalization of the current techniques to massive noninteracting theories as well as to multipoint correlators. The appendix contains information on elementary mathematical aspects of $q$-series relevant to proper analysis of twist-antitwist functions.

\section{TWIST FIELDS AND THEIR CORRELATORS}

The field theoretical definition of the twist field $V_{\alpha}$ was proposed in Refs. $[16,20]$ within the framework of the twodimensional Ising model as a continuum analogue of lattice (dis)order operators [21]. They were further generalized within the formalism of the path integral to field models with scalars and fermions [22,23], where $V_{\alpha}$ was shown to be defined by nonpolynomial and not manifestly local composite operators built up from elementary quantum fields, namely,

$$
V_{\alpha}(z)=\exp \left(2 \pi i \alpha \int_{C_{[z, \infty]}} d z_{\mu}^{\prime} \varepsilon_{\mu \nu} j_{\nu}\left(z^{\prime}\right)\right)
$$

Here the integral runs along an arbitrary contour $C_{[x, \infty]}$ from the position of the operator insertion to infinity with the integrand determined by the U(1) current, which for the complex scalar reads

$$
j_{\mu}=\left(\partial_{\mu} \phi^{*}\right) \phi-\phi^{*}\left(\partial_{\mu} \phi\right) .
$$

Its conservation implies the path independence of the definition (2.1),

$$
\int_{C_{[z, \infty]}} d z_{\mu}^{\prime} \varepsilon_{\mu \nu} j_{\nu}\left(z^{\prime}\right)=\int_{S} d^{2} z^{\prime} \partial_{\mu} j_{\mu}\left(z^{\prime}\right)+\int_{C_{[z, \infty]}^{\prime}} d z_{\mu}^{\prime} \varepsilon_{\mu \nu} j_{\nu}\left(z^{\prime}\right)
$$

where the first term vanishes upon the application of the Stokes theorem with $\partial S=C \cup\left(-C^{\prime}\right)$. In the above equation, it is assumed that $0<\alpha<1$, which is all one needs for calculation of branch point twist fields from $\alpha=k / N$ and $0 \leq k<N$.

As was demonstrated in Ref. [22], the correlation functions of twist fields in two-dimensional quantum field theory can be reformulated as statistical mechanics of matter fields in the external field of Dirac strings (or Aharonov-Bohm vortices after a gauge transformation). Namely, it is given by the functional integral

$$
\begin{aligned}
\left\langle\prod_{j} V_{\alpha_{j}}\left(z_{j}\right)\right\rangle= & \int\left[D \phi D \phi^{*}\right] \exp \left(-\int d^{2} z\left|D_{\mu} \phi(z)\right|^{2}\right) / \\
& \int\left[D \phi D \phi^{*}\right] \exp \left(-\int d^{2} z\left|\partial_{\mu} \phi(z)\right|^{2}\right),
\end{aligned}
$$

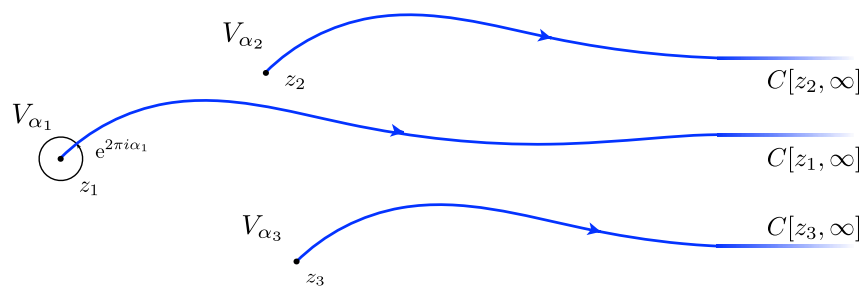

FIG. 1. Generic correlation function of twist operators at insertion points $z_{j}$ and discontinuity contours $C\left[z_{j}, \infty\right]$, with eigenfunctions of the corresponding Laplace equation developing twisted periodicity conditions (2.9).

where the gauge field in the covariant derivative $D_{\mu}=$ $\partial_{\mu}-i A_{\mu}(z)$ is determined by the potential

$$
A_{\mu}(z)=2 \pi \sum_{j} \alpha_{j} \int_{\left[z_{j}, \infty\right)} d z_{\nu}^{\prime} \varepsilon_{\nu \mu} \delta^{(2)}\left(z^{\prime}-z\right) .
$$

See Fig. 1 for a graphical representation.

The path independence in the definition of the twist fields is traded off for the gauge invariance of the path integral. Then, making use of this freedom, we can choose all the paths as straight lines aligned with the $x$ axis. Without lost of generality, we place all operators along the $x$ axis, such that the vector potential is

$A_{x}(z)=0, \quad A_{y}(z)=2 \pi \delta\left(z_{y}\right) \sum_{i} \alpha_{i} \theta\left(z_{x}-x_{j}\right)$,

where the components of any two-vector are $z_{\mu}=\left(z_{x}, z_{y}\right)$. Performing a gauge transformation, the external field takes on a form of a collection of the Aharonov-Bohm fluxes,

$$
A_{\mu}(z)=\varepsilon_{\nu \mu} \sum_{j} \alpha_{j} \frac{\left(z-z_{j}\right)_{\nu}}{\left(z-z_{j}\right)^{2}}
$$

We do not use this form in the current paper though.

The resulting path integrals for the correlation function (2.3) being Gaussian in nature can immediately be evaluated and yield

$$
\left\langle\prod_{j} V_{\alpha_{j}}\left(z_{j}\right)\right\rangle=\frac{\operatorname{det} \Delta_{0,0, \ldots}}{\operatorname{det} \Delta_{\alpha_{1}, \alpha_{2}, \ldots}},
$$

with $\Delta_{\alpha_{1}, \alpha_{2}, \ldots}=\left|D_{\mu}\right|^{2}$. The problem is thus reformulated as a spectral problem for the Laplace operator in the external field of Dirac strings (2.5). Since the latter field exists only on the $x$ axis, one solves the free-space Laplace eigenvalue equation

$$
\Delta_{0,0, \ldots} \Phi(z)=-E^{2} \Phi(z), \quad z \notin \cup_{j}\left[z_{j}, \infty\right),
$$

with eigenfunctions possessing nontrivial monodromies around each point $z_{j}$, 


$$
\Phi\left(z_{\mho_{j}}\right)=\mathrm{e}^{2 \pi i \alpha_{j}} \Phi(z),
$$

with $z_{\circlearrowleft_{j}}$ denoting a $2 \pi$ rotation of $z=z_{x}+i z_{y}$ around $z_{j}$; see Fig. 1.

Equivalently, the spectral problem can be viewed as the one for a Laplacian on Euclidean space with conical points $\left\{z_{j}\right\}$. One peculiarity of this is that a choice has to be made in order to make it a self-adjoint operator. There are infinitely many possibilities to achieve it and they are driven by the prescription of a particular asymptotic behavior near the conical points from the functional space of the Laplacian. In the current paper, we consider Friedrichs extension, which, in physical terms, merely implies that its eigenfunctions are bounded near the conical points; see, e.g., Ref. [24] for a comprehensive discussion. For the gauge transformed configuration (2.6), it means that the Aharonov-Bohm vortices are impenetrable and, thus, the eigenfunctions of the Laplace operator vanish there. Let us point out that other self-adjoint extensions were discussed in Refs. [25,26].

In order to properly calculate the determinants, we employ the zeta regularization $[27,28]$ such that

$$
\operatorname{det} \Delta=\exp \left(-\mathcal{Z}^{\prime}(0)\right)
$$

and the zeta function

$$
\mathcal{Z}(s)=\sum_{n} E_{n}^{-2 s}
$$

sums up all strictly positive eigenvalues of the spectral problem
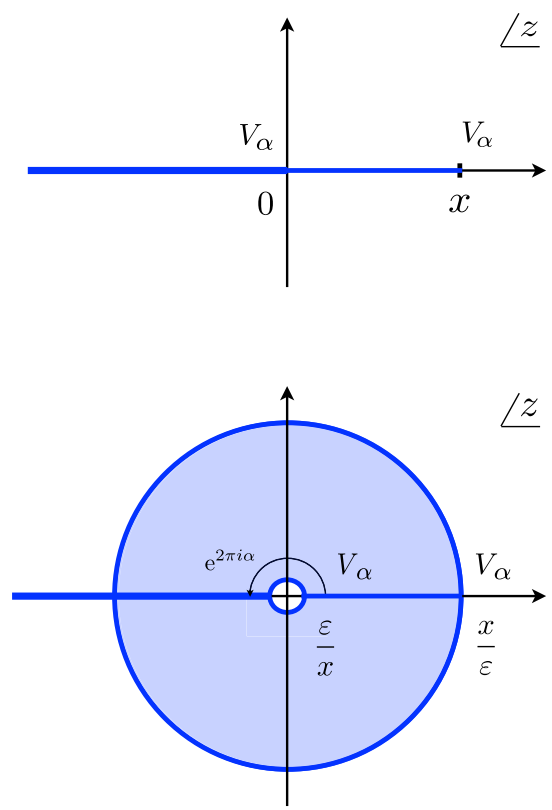

FIG. 2. Cut structure from the insertion of twist-twist (left panel) and twist-antitwist operators (right panel) in the complex plane. Below each case is a graphical representation under the regularized form of the map (3.2), when the twist operator at $z=x$ moves to infinity and explicitly exhibited twisted periodicity conditions.

$$
\Delta \Phi_{n}=-E_{n}^{2} \Phi_{n}
$$

It is regular in the vicinity of $s=0$. Recently, this technique was used to compute the vacuum expectation value of twist fields on a disk [29] and the explicit normalization coefficient naturally arises from that calculation. In the next two sections, we extend the corresponding consideration to two-point functions.

\section{MASSLESS TWIST-TWIST CORRELATOR}

Let us start our analysis with the two-point correlation function of twist operators in free massless theory. According to our previous discussion it is given by the ratio of determinants of Laplace operators in free space and in the presence of two Dirac strings,

$$
\left\langle V_{\alpha}^{\mathrm{s}}(0) V_{\alpha}^{\mathrm{s}}(x)\right\rangle=\frac{\operatorname{det} \Delta_{0,0}}{\operatorname{det} \Delta_{\alpha, \alpha}} .
$$

Making use of the path independence of twist operators, their contours are chosen as in Fig. 2 (top left panel). The coordinate transformation

$$
z_{x} \rightarrow \frac{z_{x}}{x-z_{x}}
$$

leaves the twist operator at $z_{x}=0$ intact, while moving the one at $z_{x}=x$ to infinity. This map eliminates all scales from the problem, however. Therefore, one has to tread more carefully and introduce a regulator by cutting out an infinitesimal $\varepsilon$-vicinity around insertions before performing (3.2), i.e., take $\left|z_{x}\right| \geq \varepsilon$ and $\left|z_{x}-x\right| \geq \varepsilon$. The resulting
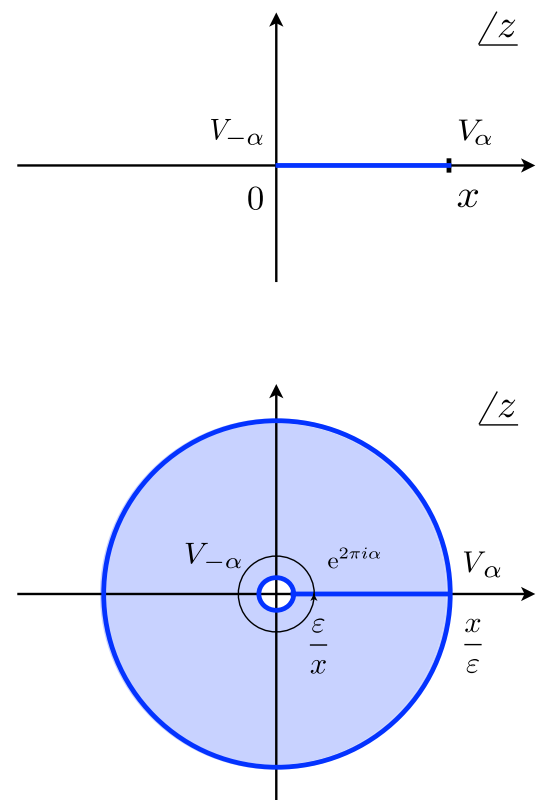
geometry takes on the form shown in Fig. 2 (bottom left panel).

To compute the determinant of the Laplacian $\Delta_{\alpha, \alpha}$, we have to solve the corresponding spectral problem. In polar coordinates, $z_{x}+i z_{y}=r \mathrm{e}^{i \vartheta}$, the equation admits the form

$$
\left(\frac{\partial^{2}}{\partial(\ln r)^{2}}+\frac{\partial^{2}}{\partial \vartheta^{2}}\right) \Phi(r, \vartheta)=-E^{2} r^{2} \Phi(r, \vartheta) .
$$

Separating the variables

$$
\Phi(r, \theta)=R(r) \Theta(\vartheta), \quad R(r)=J_{|\nu|}(E r), \quad \Theta(\vartheta)=\mathrm{e}^{i \nu \vartheta},
$$

the twisted periodicity condition on the angular function $\Theta(\pi)=\mathrm{e}^{2 \pi i \alpha} \Theta(0)$ provides the quantization condition for $\nu=2 m+2 \alpha$ with integer $m=0, \pm 1, \pm 2, \ldots$ The requirement of the bounded behavior at the position of the twist fields fixes the radial wave function to the Bessel function of the first kind and yields the eigenvalues

$$
E_{m, n}^{(\alpha)}=j_{|2 m+2 \alpha|, n} /(x / \varepsilon),
$$

in terms of the positive roots $j_{|2 m+2 \alpha|, n}$ of $J_{|2 m+2 \alpha|}$. Making use of the zeta function regularization, we can rewrite this as

$$
\begin{aligned}
\ln \frac{\operatorname{det} \Delta_{0,0}}{\operatorname{det} \Delta_{\alpha, \alpha}}= & \mathcal{Z}_{1}^{\prime}(0, \alpha)+\mathcal{Z}_{2}^{\prime}(0, \alpha)+\mathcal{Z}_{2}^{\prime}(0,-\alpha) \\
& -\mathcal{Z}_{1}^{\prime}(0,0)-2 \mathcal{Z}_{2}^{\prime}(0,0)
\end{aligned}
$$

where the prime stands for the derivative with respect to the first argument and we introduced one- and two-dimensional Bessel zeta functions,

$$
\mathcal{Z}_{1}(s, \alpha)=\sum_{n=1}^{\infty}\left(E_{0, n}^{(\alpha)}\right)^{-2 s}, \quad \mathcal{Z}_{2}(s, \alpha)=\sum_{m=1}^{\infty} \sum_{n=1}^{\infty}\left(E_{m, n}^{(\alpha)}\right)^{-2 s} .
$$

\section{A. One-dimensional contribution}

To start with, let us analyze the one-dimensional contribution. In fact, we do not even have to introduce $\mathcal{Z}_{1}$ as the determinant for the $m=0$ case can be easily deduced from the application of the Gelfand-Yaglom theorem [30] (see Ref. [31] for a comprehensive introduction) since the problem is reduced to the one-dimensional radial problem. Let us start with this simple calculation and regularize the Laplacian with a mass $M, \Delta_{\alpha, \alpha} \rightarrow \Delta_{\alpha, \alpha}+M^{2}$, such that a regular solution at the origin $\Psi_{\alpha}(r)=J_{2 \alpha}(M r)$ of the initial value problem

$$
\left.\left(\Delta_{\alpha, \alpha}+M^{2}\right)\right|_{m=0} \Psi_{\alpha}(r)=0
$$

provides the value of the regularized determinant,

$$
\left.\operatorname{det}\left(\Delta_{\alpha, \alpha}+M^{2}\right)\right|_{m=0}=\Psi_{\alpha}(x / \varepsilon) .
$$

Multiplying the right-hand side by the renormalization factor and taking the limit $M \rightarrow 0$, we immediately find

$\frac{\left.\operatorname{det} \Delta_{0}\right|_{m=0}}{\left.\operatorname{det} \Delta_{\alpha}\right|_{m=0}}=\lim _{M \rightarrow 0} \frac{M^{2 \alpha} \Psi_{0}(x / \varepsilon)}{\Psi_{\alpha}(x / \varepsilon)}=2^{2 \alpha} \Gamma(1+2 \alpha)\left(\frac{\varepsilon}{x}\right)^{2 \alpha}$.

To verify this result, we now repeat it within the formalism of the zeta function regularization. To avoid dealing with the Bessel spectrum, $\mathcal{Z}_{1}$ is rewritten in terms of the resolvent

$$
\mathcal{Z}_{1}(s, \alpha)=\int_{C^{+}} \frac{d \sigma}{2 \pi i} \sigma^{-s} \mathcal{R}_{1}(\sigma, \alpha)
$$

over a Hankel-like contour $C^{+}$running counterclockwise to the left of all poles of the integrand but to the right of the origin. Here $\mathcal{R}_{1}(\sigma, \alpha)=-\mathcal{U}_{1}^{\prime}(\sigma, \alpha)$ is traded for the potential $\mathcal{U}_{1}$,

$$
\begin{aligned}
\mathcal{U}_{1}(\sigma, \alpha)= & -\ln \prod_{n=1}^{\infty}\left(1+\left(\frac{\sqrt{-\sigma}}{j_{2 \alpha, n}} \frac{x}{\varepsilon}\right)^{2}\right) \\
= & -\ln I_{2 \alpha}\left(\frac{\sqrt{-\sigma} x}{\varepsilon}\right)+2 \alpha \ln \frac{\sqrt{-\sigma} x}{2 \varepsilon} \\
& -\ln \Gamma(1+2 \alpha),
\end{aligned}
$$

and we employed the classic infinite series representation of the modified Bessel function $I_{2 \alpha}$ due to Watson [32]. The function $\mathcal{U}_{1}$ vanishes at the origin $\sigma=0$. It admits a systematic expansion in terms of powers and logarithms of $\sigma[33,34]$. In fact, the constant and $\log (-\sigma)$ term in its expansion determine the leading two terms in the small-s expansion of the Bessel zeta function. Moving the derivative off $\mathcal{U}_{1}$ onto the function to its left and exponentiating $\sigma^{-s}$ by means of the Schwinger formula, we find

$\Gamma(s) \mathcal{Z}_{1}(s, \alpha)=-s \int_{0}^{1} d t t^{s-1} \int_{C^{-}} \frac{d \sigma}{2 \pi i} \frac{\mathrm{e}^{-t \sigma}}{\sigma} \mathcal{U}_{1}(\sigma, \alpha)+O(s)$.

Here, we deformed the integration contour $C^{+}$to the left of the origin and, since $\mathcal{U}_{1}(0, \alpha)=0$, the $C^{-}$becomes the true Hankel contour. We also restricted the integration with respect to the Schwinger time over the half line to the interval $[0,1]$ which holds up to higher order powers of $s$. The large- $\sigma$ asymptotics of the potential reads

$$
\mathcal{U}_{1}(\sigma, \alpha)=\mathcal{U}_{1,1}(\alpha) \ln (-\sigma)+\mathcal{U}_{1,0}(\alpha)+\cdots,
$$


with

$\mathcal{U}_{1,1}(\alpha)=\frac{1}{4}+\alpha$

$\mathcal{U}_{1,0}(\alpha)=2\left(\frac{1}{4}+\alpha\right) \ln \frac{x}{\varepsilon}-\ln \frac{2^{2 \alpha-1 / 2} \Gamma(1+2 \alpha)}{\sqrt{\pi}}$.

Evaluating the $\sigma$ and $t$ integrals, we find

$\Gamma(s) \mathcal{Z}_{1}(s, \alpha)=\mathcal{U}_{1,1}(\alpha) \gamma_{\mathrm{E}}-\mathcal{U}_{1,0}(\alpha)-\frac{1}{s} \mathcal{U}_{1,1}(\alpha)+O(s)$,

such that the expansion in $s$ yields

$\mathcal{Z}_{1}^{\prime}(0, \alpha)=-2\left(\frac{1}{4}+\alpha\right) \ln \frac{x}{\varepsilon}+\ln \frac{2^{2 \alpha-1 / 2} \Gamma(1+2 \alpha)}{\sqrt{\pi}}$,

and coincides with Eq. (3.10) obtained with the help of the Gelfand-Yaglom theorem and mass regularization.

\section{B. Two-dimensional contribution}

Turning to the two-dimensional Bessel zeta function, we cast $\mathcal{Z}_{2}$ into the form

$\mathcal{Z}_{2}(s, \alpha)=-s \sum_{m=1}^{\infty}(m+\alpha)^{-2 s} \int_{C^{+}} \frac{d \sigma}{2 \pi i} \sigma^{-s-1} \mathcal{U}_{2}(\sigma, m+\alpha)$,

with the potential

$$
\begin{aligned}
\mathcal{U}_{2}(\sigma, m+\alpha)= & -\ln \prod_{n=1}^{\infty}\left(1+\left(\frac{(m+\alpha) \sqrt{-\sigma} x}{j_{2 m+2 \alpha, n}}\right)^{2}\right) \\
= & -\ln I_{2 m+2 \alpha}\left((m+\alpha) \frac{\sqrt{-\sigma} x}{\varepsilon}\right) \\
& +2(m+\alpha) \ln \frac{(m+\alpha) \sqrt{-\sigma} x}{2 \varepsilon} \\
& -\ln \Gamma(1+2 m+2 \alpha) .
\end{aligned}
$$

The calculation of the Kronecker limit formula for the double Bessel zeta function follows the footsteps of Ref. [29] for twist operator on a disk. Since the sum accompanying the factor of $s$ in Eq. (3.18) is not analytic in $s$ in the vicinity of $s=0$, we split $\mathcal{U}_{2}$ into two contributions,

$\mathcal{U}_{2}(\sigma, m+\alpha)=\mathcal{V}_{2}(\sigma, m+\alpha)+\frac{1}{m+\alpha} \mathcal{W}_{2}(\sigma)$

Notice that only the second term can generate a pole at $s=0$. The accompanying function $\mathcal{W}_{2}$ can be found from the uniform expansion of the Bessel function [35],
$I_{\nu}(\nu z) \stackrel{\nu \rightarrow \infty}{=} \frac{\mathrm{e}^{\nu\left[\sqrt{1+z^{2}}+\ln z /\left(1+\sqrt{1+z^{2}}\right)\right]}}{\sqrt{2 \pi \nu}\left(1+z^{2}\right)^{1 / 4}}\left[1+\frac{1}{\nu} U_{1}(z)+O\left(\nu^{-2}\right)\right]$,

where

$$
U_{1}(z)=\frac{1}{8}\left(1+z^{2}\right)^{-1 / 2}-\frac{5}{24}\left(1+z^{2}\right)^{-3 / 2},
$$

such that

$$
\mathcal{W}_{2}(\sigma)=-\frac{1}{2} U_{1}\left(\frac{\sqrt{-\sigma} x}{2 \varepsilon}\right)
$$

The calculation of the integral associated with this contribution can be done immediately with the result

$\int_{C^{+}} \frac{d \sigma}{2 \pi i} \sigma^{-s-1} \mathcal{W}_{2}(\sigma)=-\frac{\Gamma\left(s+\frac{1}{2}\right)}{24 \Gamma(s) \Gamma\left(\frac{1}{2}\right)}\left(\frac{1}{s}+5\right)\left(\frac{x}{2 \varepsilon}\right)^{2 s}$.

The integral with $\mathcal{V}_{2}$ is evaluated analogously to the onedimensional case, namely,

$$
\begin{gathered}
\Gamma(s+1) \int_{C^{+}} \frac{d \sigma}{2 \pi i} \sigma^{-s-1} \mathcal{V}_{2}(\sigma, m+\alpha)=-\mathcal{V}_{2}(0, m+\alpha) \\
-\left(\gamma_{\mathrm{E}}-\frac{1}{s}\right) \mathcal{V}_{2,1}(m+\alpha)+\mathcal{V}_{2,0}(m+\alpha)+O(s)
\end{gathered}
$$

where $\mathcal{V}_{2, n}$ are extracted from the large- $\sigma$ expansion

$\mathcal{V}_{2}(\sigma, m+\alpha)=\mathcal{V}_{2,1}(m+\alpha) \ln (-\sigma)+\mathcal{V}_{2,0}(m+\alpha)+\cdots$,

with

$\mathcal{V}_{2,1}(m+\alpha)=\frac{1}{4}+m+\alpha$

$$
\begin{aligned}
\mathcal{V}_{2,1}(m+\alpha)= & 2\left(\frac{1}{4}+m+\alpha\right) \ln \left((m+\alpha) \frac{x}{\varepsilon}\right) \\
& -\frac{2^{2 m+2 \alpha-1 / 2} \Gamma(1+2 m+2 \alpha)}{\sqrt{\pi}} .
\end{aligned}
$$

Putting these together, we obtain 


$$
\begin{aligned}
\Gamma(s) \mathcal{Z}_{2}(s, \alpha)= & \sum_{m=1}^{\infty}(m+\alpha)^{-2 s}\left[\mathcal{V}_{2}(0, m+\alpha)\right. \\
& \left.+\left(\gamma_{\mathrm{E}}-\frac{1}{s}\right) \mathcal{V}_{2,1}(m+\alpha)-\mathcal{V}_{2,0}(m+\alpha)\right] \\
& +(1+5 s) \frac{\Gamma\left(s+\frac{1}{2}\right)}{24 \Gamma\left(\frac{1}{2}\right)}\left(\frac{x}{2 \varepsilon}\right)^{2 s} \\
& \times \sum_{m=1}^{\infty}(m+\alpha)^{-2 s-1}+O(s) .
\end{aligned}
$$

Performing the sums, the double zeta functions admit the form

$$
\begin{aligned}
\Gamma(s) \mathcal{Z}_{2}(s, \alpha)= & 2 \zeta^{\prime}(2 s-1,1+\alpha)+\frac{1}{2} \zeta^{\prime}(2 s, 1+\alpha) \\
& -\frac{1}{2} \ln (4 \pi) \zeta(2 s, 1+\alpha)+\left(\gamma_{\mathrm{E}}-\frac{1}{s}-2 \ln \frac{x}{2 \varepsilon}\right) \\
& \times\left[\zeta(2 s-1,1+\alpha)+\frac{1}{4} \zeta(2 s, 1+\alpha)\right] \\
& +\frac{\Gamma\left(s+\frac{1}{2}\right)}{24 \Gamma\left(\frac{1}{2}\right)}(1+5 s) \zeta(2 s+1,1+\alpha)\left(\frac{x}{2 \varepsilon}\right)^{2 s} \\
& +\chi_{2}(s, \alpha)+O(s),
\end{aligned}
$$

in terms of the Hurwitz zeta functions and the function

$$
\begin{aligned}
\chi_{2}(s, \alpha)= & \sum_{m=1}^{\infty}(m+\alpha)^{-2 s} \ln \Gamma(1+2 m+2 \alpha) \\
& -\frac{1}{24} \zeta(2 s+1,1+\alpha)
\end{aligned}
$$

analytic at the origin $s=0$. For the two-point twist-twist correlator (3.1), we get

$$
\left\langle V_{\alpha}^{\mathrm{s}}(0) V_{\alpha}^{\mathrm{s}}(x)\right\rangle=c_{\alpha, \alpha}^{\mathrm{s}}\left(\frac{\varepsilon}{x}\right)^{2 h_{\alpha}^{\mathrm{s}}},
$$

with the normalization constant being

$$
\begin{aligned}
c_{\alpha, \alpha}^{\mathrm{s}}= & 4^{\alpha(1-\alpha)} \Gamma(1+2 \alpha) \exp \left(\chi_{2}(0, \alpha)+\chi_{2}(0,-\alpha)\right. \\
& \left.-2 \chi_{2}(0,0)-\frac{1}{24}\left[\psi(1-\alpha)+\psi(1+\alpha)+2 \gamma_{\mathrm{E}}\right]\right),
\end{aligned}
$$

and the well-known scaling dimension

$$
h_{\alpha}^{\mathrm{s}}=\alpha(1-\alpha) \text {, }
$$

of the scalar twist field [1,2].

\section{MASSLESS TWIST-ANTITWIST CORRELATOR}

We turn now to the twist-antitwist correlator, determined through the ratio

$$
\left\langle V_{-\alpha}^{\mathrm{s}}(0) V_{\alpha}^{\mathrm{s}}(x)\right\rangle=\frac{\operatorname{det} \Delta_{0,0}}{\operatorname{det} \Delta_{-\alpha, \alpha}} .
$$

Now, compared to the just analyzed two-point function, the cut does not extend past the position of the (anti)twist fields; see the right panel in Fig. 2. Therefore, one can use the conformal map $\varrho=\ln r$ from the annulus $\{\varepsilon / x \leq r \leq x / \varepsilon$, $0 \leq \vartheta \leq 2 \pi\}$ to the cylinder $\left\{-\varrho_{0} \leq \varrho \leq \varrho_{0}, 0 \leq \vartheta \leq 2 \pi\right\}$ with $\varrho_{0} \equiv \ln (x / \varepsilon)$. It changes the metric by an overall factor $d r^{2}+r^{2} d \vartheta^{2}=\mathrm{e}^{2 \varrho}\left(d Q^{2}+d \vartheta^{2}\right)$ and allows one to rewrite the determinant on the annulus in terms of the one on the cylinder making use of the Polyakov formula [36-38],

$$
\left.\ln \operatorname{det} \Delta\right|_{\mathrm{ann}}=\left.\ln \operatorname{det} \Delta\right|_{\mathrm{cyl}}-\frac{1}{3} \varrho_{0} .
$$

The Jacobian however cancels between the vortex-dependent $\Delta_{-\alpha, \alpha}$ and the free $\Delta_{0,0}$ Laplacians. The eigenspectrum equation on the cylinder is

$$
\left(\frac{\partial^{2}}{\partial \varrho^{2}}+\frac{\partial^{2}}{\partial \vartheta^{2}}\right) \Phi(\varrho, \vartheta)=-E^{2} \Phi(\varrho, \vartheta),
$$

with eigenfunctions in the separated variables being

$$
\begin{aligned}
\Phi(\varrho, \vartheta) & =R(\varrho) \Theta(\vartheta), \quad R(\varrho)=\sin \left(K\left(\varrho-\varrho_{0}\right)\right), \\
\Theta(\vartheta) & =\mathrm{e}^{i \nu \vartheta}
\end{aligned}
$$

obeying the boundary conditions, $R\left(\varrho_{0}\right)=R\left(-\varrho_{0}\right)=0$ and $\Theta(2 \pi)=\mathrm{e}^{2 \pi i \alpha} \Theta(0)$. The latter provide the quantization conditions for the integration constants

$$
\nu=m+\alpha, \quad K=\kappa n, \quad \kappa=\frac{\pi}{2 \varrho_{0}},
$$

with integer $0<n,-\infty<m<\infty$ and omission of the $m=0$, for which the wave function vanishes identically. The eigenspectrum is thus

$$
E_{n, m}^{(\alpha)}=\sqrt{(m+\alpha)^{2}+(\kappa n)^{2}} .
$$

The correlation function of twist-antitwist operators is then determined in its terms by the infinite product

$$
\left\langle V_{-\alpha}^{\mathrm{s}}(0) V_{\alpha}^{\mathrm{s}}(x)\right\rangle=\prod_{m=-\infty}^{\infty} \prod_{n=1}^{\infty}\left(\frac{E_{n, m}^{(0)}}{E_{n, m}^{(\alpha)}}\right)^{2} .
$$

The prime on the product stands for the omission of the zero mode $m=0$. 


\section{A. From twists to branch point twists}

Due to simplicity of the spectrum (4.6), the resulting products can be analyzed directly without the use of the zeta function regularization. And one can deduce two different single-product representations which are amenable to further analysis. First, evaluating the product with respect to the index $n$ first, we obtain a representation

$$
\begin{aligned}
& \left\langle V_{-\alpha}^{\mathrm{s}}(0) V_{\alpha}^{\mathrm{s}}(x)\right\rangle \\
& \quad=\prod_{m=-\infty}^{\infty} \prod_{n=1}^{\infty} \frac{(\kappa n)^{2}+m^{2}}{(\kappa n)^{2}+(m+\alpha)^{2}} \\
& \quad=\frac{\sin (\pi \alpha)}{\pi \alpha} \prod_{m=1}^{\infty} \frac{\left(1-\mathrm{e}^{-4 m \varrho_{0}}\right)^{2}}{\left(1-\mathrm{e}^{-4(m+\alpha) \varrho_{0}}\right)\left(1-\mathrm{e}^{\left.-4(m-\alpha) \varrho_{0}\right)}\right.},
\end{aligned}
$$

where the products can be reexpressed in terms of Lambert's series. We provide, however, an equivalent representation by first calculating the product in $m$ to make connection with previous analyses. Namely, rearranging the factors as

$$
\begin{aligned}
& \prod_{m=-\infty}^{\infty} \prod_{n=1}^{\infty} \frac{(\kappa n)^{2}+m^{2}}{(\kappa n)^{2}+(m+\alpha)^{2}} \\
& =\prod_{n=1}^{\infty} \prod_{m=1}^{\infty}\left(1-\frac{\alpha^{2}}{(m-i \kappa n)^{2}}\right)^{-1}\left(1-\frac{\alpha^{2}}{(m+i \kappa n)^{2}}\right)^{-1}
\end{aligned}
$$

then making use of

$$
\prod_{m=1}^{\infty}\left(1-\frac{\alpha^{2}}{(m+\kappa)^{2}}\right)=\frac{\Gamma^{2}(1+\kappa)}{\Gamma(1-\alpha+\kappa) \Gamma(1+\alpha+\kappa)},
$$

and eventually simplifying the product by means of $\Gamma(1+Y) \Gamma(1-Y)=(\pi Y) / \sin (\pi Y)$. These manipulations immediately yield

$$
\left\langle V_{-\alpha}^{\mathrm{s}}(0) V_{\alpha}^{\mathrm{s}}(x)\right\rangle=\prod_{n=1}^{\infty} \frac{\left(1-q^{n}\right)^{2}}{\left(1-\mathrm{e}^{2 \pi i \alpha} q^{n}\right)\left(1-\mathrm{e}^{-2 \pi i \alpha} q^{n}\right)}
$$

where we introduced a compact notation

$$
q=\mathrm{e}^{2 \pi \kappa} .
$$

Since this correlator plays a distinguished role in defining the geometric entropy [6] according to Eq. (1.10), let us pass from the (anti)twist fields to branch point (anti)twist operators. We use Eq. (1.6) to find

$$
\left\langle\bar{V}^{\mathrm{s}}(0) V^{\mathrm{s}}(x)\right\rangle=\prod_{k=1}^{N-1}\left\langle V_{-k / N}(0) V_{k / N}(x)\right\rangle=\left[\prod_{n=1}^{\infty} \frac{\left(1-q^{n}\right)^{N}}{\left(1-q^{n N}\right)}\right]^{2}
$$

where we made use of the fact that

$$
\prod_{k=1}^{N-1}\left(1-Q \mathrm{e}^{ \pm 2 \pi i k / N}\right)=\frac{1-Q^{N}}{1-Q} .
$$

This was the starting point of the analysis in Ref. [4]. The infinite products in the above equation can be cast in terms of the well-studied $q$-Pochhammer symbol, see, e.g., [39],

$$
\prod_{n=1}^{\infty}\left(1-Q^{n}\right)=(Q, Q)_{\infty}
$$

such that

$$
\left\langle\bar{V}^{\mathrm{s}}(0) V^{\mathrm{s}}(x)\right\rangle=\frac{(q, q)_{\infty}^{2 N}}{\left(q^{N}, q^{N}\right)_{\infty}^{2}} .
$$

Then its ultraviolet asymptotics as $\varepsilon \rightarrow 0$ can be easily read off from the well-known behavior of the $q$-Pochhammer symbols as $q \rightarrow 1_{-}$due to Watson estimation [40,41] (see also Refs. [42] for recent accounts),

$$
(q, q)_{\infty}=\left(\frac{2 \pi}{1-q}\right)^{1 / 2} \exp \left(\frac{\zeta(2)}{\ln q}\right)[1+O(q)] .
$$

Then the two-point function on an $\mathrm{N}$-sheeted Riemann surface reads

$$
\left\langle\bar{V}^{\mathrm{s}}(0) V^{\mathrm{s}}(x)\right\rangle=N\left(\frac{\ln \frac{x}{\varepsilon}}{2 \pi}\right)^{N-1}\left(\frac{\varepsilon}{x}\right)^{\frac{1}{6}\left(N-\frac{1}{N}\right)},
$$

and acquires a multiplicative logarithmic dependence, which is typical of logarithmic CFTs [19]. Making use of the definitions (1.9) and (1.10), this fact implies the emergence of an additive $\ln \ln$-correction to the famous entanglement entropy scaling

$$
S_{\mathrm{vN}} \sim \frac{\mathrm{c}}{6} \ln \frac{x}{\varepsilon}-\ln \ln \frac{x}{\varepsilon}+\text { const. }
$$

with ultraviolet cutoff $\varepsilon$ due to Ref. [4]. Here $c=2$ is the central charge of a complex scalar studied in this work. The second term in the above equation was recently noticed by numerically resumming form factor expansion to two-point functions in Ref. [43] and via analytical methods in Ref. [44], though our sign is opposite to the one in the first study.

The asymptotic scaling of the correlation function (4.11) can be analyzed in a similar fashion. However, before we do it, in a general spirit of this paper, we provide the derivation of (4.11) within the framework of zeta function regularization in the next section. This analysis is of interest in its own right as it provides a derivation of the Kronecker limit formula $[38,45]$ for the double spectral series in question.

\section{B. Zeta function regularization}

Let us calculate the two-point correlation function (4.1) by means of the zeta function regularization. We introduce 


$$
\mathcal{Z}(s, \alpha)=\sum_{n, m=1}^{\infty}\left[(m+\alpha)^{2}+(\kappa n)^{2}\right]^{-s}
$$

which is a generalization of the Epstein zeta function [46]. As in Sec. III B, we write it as a contour integral

$$
\mathcal{Z}(s, \alpha)=-s \sum_{m=1}^{\infty}(m+\alpha)^{-2 s} \int_{C_{+}} \frac{d \sigma}{2 \pi i} \sigma^{-s-1} \mathcal{U}(\sigma, m+\alpha),
$$

in terms of the potential

$$
\begin{aligned}
\mathcal{U}(\sigma, m+\alpha) & =-\ln \prod_{n=1}^{\infty}\left(1+\frac{(m+\alpha)^{2}(-\sigma)}{(m+\alpha)^{2}+(\kappa n)^{2}}\right) \\
& =\ln (1-\sigma)+\ln \frac{\Gamma(i \sqrt{1-\sigma}(m+\alpha) / \kappa) \Gamma(-i \sqrt{1-\sigma}(m+\alpha) / \kappa)}{\Gamma(i(m+\alpha) / \kappa) \Gamma(-i(m+\alpha) / \kappa)}
\end{aligned}
$$

First, one notices that $\mathcal{U}(0, m+\alpha)=0$, so there is no contribution from the residue at $\sigma=0$ in Eq. (3.24). Second, since the argument of the logarithm is an even function of $(m+\alpha)$, the application of the Stirling formula with the first power correction accounted for, i.e., $\ln \Gamma(Y)=\cdots+1 /(12 Y)+O\left(1 / Y^{3}\right)$, implies that $\mathcal{W}=0$ in the analogue of Eq. (3.20) for the case at hand. One finds

$$
\Gamma(s+1) \int_{C^{+}} \frac{d \sigma}{2 \pi i} \sigma^{-s-1} \mathcal{U}(\sigma, m+\alpha)=-\left(\gamma_{\mathrm{E}}-\frac{1}{s}\right) \mathcal{U}_{1}(m+\alpha)+\mathcal{U}_{0}(m+\alpha)+O(s),
$$

where the coefficients $\mathcal{U}_{1 / 0}$ accompanying $\ln ^{1 / 0}(-\sigma)$ dependence in the asymptotic large- $\sigma$ expansion (3.25) are

$$
\begin{aligned}
& \mathcal{U}_{1}(m+\alpha)=\frac{1}{2}, \\
& \mathcal{U}_{0}(m+\alpha)=\ln \left(2 \sinh \frac{\pi(m+\alpha)}{\kappa}\right) .
\end{aligned}
$$

The Kronecker-like limit formula then reads

$$
\begin{aligned}
\Gamma(s) \mathcal{Z}(s, \alpha)= & \sum_{m=1}^{\infty}(m+\alpha)^{-2 s}\left[\left(\gamma_{\mathrm{E}}-\frac{1}{s}\right) \mathcal{U}_{1}(m+\alpha)\right. \\
& \left.-\mathcal{U}_{0}(m+\alpha)\right]+O(s) .
\end{aligned}
$$

In its terms, the correlation function is

$\ln \left\langle V_{-\alpha}^{\mathrm{s}}(0) V_{\alpha}^{\mathrm{s}}(x)\right\rangle=\mathcal{Z}^{\prime}(0, \alpha)+\mathcal{Z}^{\prime}(0,-\alpha)-2 \mathcal{Z}^{\prime}(0,0)$,

and can immediately be found to coincide with Eq. (4.8).

Let us conclude this section with the ultraviolet asymptotics of twist-antitwist correlator as $\varepsilon \rightarrow 0$. Making use of expansion formulas as $q \rightarrow 1_{-}$derived in the Appendix, we find the logarithmically enhanced behavior

$$
\left\langle V_{-\alpha}^{\mathrm{s}}(0) V_{\alpha}^{\mathrm{s}}(x)\right\rangle=c_{-\alpha, \alpha}^{\mathrm{s}}\left(\frac{\varepsilon}{x}\right)^{2 h_{\alpha}^{\mathrm{s}}} \ln \frac{x}{\varepsilon},
$$

with the normalization constant

$$
c_{-\alpha, \alpha}^{\mathrm{s}}=\frac{4 \sin (\pi \alpha)}{\pi} .
$$

It is important to realize that the logarithms stem from the determinant of the free Laplacian $\Delta_{0,0}$, i.e., the numerator in Eq. (4.1), providing proper normalization for the correlation function in question. The result of Ref. [44] obtained within the formalism of the angular quantization [47] agrees with this expression.

\section{INSTEAD OF A CONCLUSION}

In the main body of this paper, we focused on two-point twist-(anti)twist correlations for a massless free scalar. The massive case can be analyzed in a similar manner. To provide a sketch of the treatment, we limit ourselves to the expectation value of twist field. The solutions $\Phi(r, \vartheta)$ to the eigenvalue equation with a singe twist, decaying at infinity as $\Phi(r \rightarrow \infty, \vartheta) \sim \mathrm{e}^{-M r}$, admit the form (when cast in a contour integral form)

$$
\Phi(r, \vartheta)=\int d \kappa c(\kappa) K_{i \kappa}(r) \mathrm{e}^{-\kappa \vartheta} .
$$

It endows the system with a discrete spectrum by imposing quasiperiodicity conditions on the angular dependence and the Dirichlet boundary condition $\Phi(r=\varepsilon, \vartheta)=0$ on the circular domain $r=\varepsilon$ in the vicinity of the vortex $V_{\alpha}(0)$. The vacuum average of the scalar twist field then reads 


$$
\begin{aligned}
\ln \left\langle V_{\alpha}^{\mathrm{S}}\right\rangle_{M}= & \sum_{n=1}^{\infty} \ln \frac{\left(E_{0, n}^{(0)}\right)^{2}+M^{2}}{\left(E_{0, n}^{(\alpha)}\right)^{2}+M^{2}} \\
& +\sum_{m=1}^{\infty} \sum_{n=1}^{\infty} \ln \frac{\left(E_{m, n}^{(0)}\right)^{2}+M^{2}}{\left(E_{m, n}^{(\alpha)}\right)^{2}+M^{2}} \frac{\left(E_{m, n}^{(0)}\right)^{2}+M^{2}}{\left(E_{m, n}^{(-\alpha)}\right)^{2}+M^{2}}
\end{aligned}
$$

where as in the massless case, we separated the $m=0$ eigenvalue. According to Ref. [22], the second term involving the double sum can be related to the determinant of the Dirac operator with the Atiyah-Patodi-Singer spectral boundary conditions $[22,48,49]$ and, bosonizing the fermion, it can then be reduced to the analysis of the vacuum average of a vertex operator in the dual picture of the massive sine-Gordon model [47]. Presently, we do not address this term within the zeta function regularization, but the corresponding inhomogeneous double spectral series is of interest in its own right and requires a separate study. Here, we merely provide the result for the single spectral series associated with the $m=0$ term since it is responsible for logarithmic dependence of the vacuum expectation value in ultraviolet cutoff $\varepsilon$. The use of the Gelfand-Yaglom theorem immediately yields the following expression:

$$
\prod_{n=1}^{\infty} \frac{\left(E_{0, n}^{(0)}\right)^{2}+M^{2}}{\left(E_{0, n}^{(\alpha)}\right)^{2}+M^{2}}=\frac{K_{0}(M \varepsilon)}{K_{\alpha}(M \varepsilon)} \stackrel{\varepsilon \rightarrow 0}{\simeq} \frac{(M \varepsilon)^{\alpha}}{2^{\alpha-1} \Gamma(\alpha)} \ln \left(\frac{2 \mathrm{e}^{-\gamma_{\mathrm{E}}}}{M \varepsilon}\right) .
$$

In complete analogy to the massless case, discussed in Ref. [29], the double product develops $(M \varepsilon)^{-\alpha^{2}}$ dependence on ultraviolet cutoff completing the exponent of the $M$-dependence to the conformal dimension of the scalar twist field $h_{\alpha}$. The resulting functional dependence on the parameter $M \varepsilon$ is in agreement with previous studies, where the logarithmic corrections due to conformal symmetry breaking by mass perturbations were observed from analyses of the Painlevé equation [20,50,51], form factor resummation [43] and angular quantization [44].

The ratio of determinants defining correlators in question, also known as the relative spectral determinants [52], can be equivalently (and more efficiently) studied in noncompact space by means of the Lifshitz-Krein trace formula [53,54]. For a function $f$ of a self-adjoint operator $A_{0}$ and its perturbation $A$, with their difference $A-A_{0}$ being of the trace class,

$$
\operatorname{tr}\left[f(A)-f\left(A_{0}\right)\right]=\int d \kappa f\left(E_{\kappa}\right) \partial_{\kappa} \xi(\kappa)
$$

Here, the spectral shift function $\xi(E)$, associated with the pair $\left(A_{0}, A\right)$, is given by the Birman-Krein formula [55]

$$
\xi(\kappa)=\frac{1}{2 \pi i} \ln \operatorname{det} S(\kappa)
$$

in terms of the scattering matrix $S(E)$ on the perturbation $A-A_{0}$ (for a review, see [56]). Then, taking the function $f$ in the form of the heat kernel $f(A)=\exp (-t A)$, the vacuum expectation is given by the relative determinant regularized by means of the zeta function [52],

$$
\ln \left\langle V_{\alpha}(0)\right\rangle=-\mathcal{Z}^{\prime}\left(0, \Delta_{\alpha}, \Delta_{0}\right),
$$

where

$$
\mathcal{Z}\left(s, \Delta_{\alpha}, \Delta_{0}\right)=\int \frac{d \kappa}{2 \pi i} E_{\kappa}^{-s} \partial_{\kappa} \ln \operatorname{det} S(\kappa) .
$$

The scattering matrix on one vortex can be easily found from the asymptotic behavior of the eigenfunction (5.1) in the vicinity of the vortex $r=0$ as a ratio of coefficient between left and right movers,

$$
\Phi(r \rightarrow 0, \vartheta) \sim(r / 2)^{-i \kappa}+S(\kappa)(r / 2)^{i \kappa},
$$

with

$$
S(\kappa)=\frac{\Gamma(1-i \kappa)}{\Gamma(1+i \kappa)}
$$

With this input and explicit form of the resummed energy spectrum, see Eq. (4.11) above, one can immediately see that the expectation value (5.6) is the same as obtained with the formalism of the angular quantization advocated in Ref. [47] for fermions and recently applied to scalars in Ref. [44].

In light of the representation (5.7), one could find it instructive to explore a connection to scattering matrices on Aharonov-Bohm vortices (2.6) as a tool to compute multitwist correlators. Recently, scattering amplitudes on two fluxes were found explicitly as a solution to Painlevé V equations [57]. This formulation allows for an extension to systems with multiple vortices, i.e., surfaces with multiple conical singularities [25], and it provides a natural geometric interpretation to the gluing of conical manifolds [26] via analytic surgery procedure of Burghelea et al. [58] by interpreting the S-matrix as a kind of limiting Dirichlet-toNeumann operator. The application of these considerations to the current setup of correlation functions of twist operators will be discussed elsewhere.

\section{ACKNOWLEDGMENTS}

This work was completed during the author's visit to ENS (Paris) and IPhT (Saclay). We thank Benjamin Basso and Gregory Korchemsky for the warm hospitality at the respective institutions, and Leonid Friedlander, Ivan Kostov, Didina Serban and Frank Wilczek for discussions. This research was supported by the U.S. National Science Foundation under Grant No. PHY-1403891. 


\section{APPENDIX: ASYMPTOTIC EXPANSION}

Let us calculate the asymptotic expansion of the product

$$
\prod_{k=1}^{\infty}\left(1-a q^{k}\right)=\frac{(a, q)_{\infty}}{1-a}
$$

contributing to the cylinder partition function in the $q \rightarrow 1_{-}$ limit and $a \neq 1$. This can be done by elementary methods. The first step consists in exponentiating the terms in the product and expanding the logarithm in the region of its convergence in the Taylor expansion and then exchanging the two sums. This yields

$$
\prod_{k=1}^{\infty}\left(1-a q^{k}\right)=\exp \left(\sum_{\ell=1}^{\infty} \frac{1}{\ell} \frac{a^{\ell}}{1-q^{-\ell}}\right)
$$

Then adopting the second representation, the $q \rightarrow 1_{-}$ expansion can be easily performed with the following chain of transformations:

$$
\begin{aligned}
\ln \prod_{k=1}^{\infty}\left(1-a q^{k}\right) & =\sum_{\ell=1}^{\infty} \frac{a^{\ell}}{\ell}\left(1-\sum_{n=0}^{\infty} \frac{\ell^{n}}{n !} \ln ^{n} \frac{1}{q}\right)^{-1} \\
& =-\frac{1}{\ln \frac{1}{q}} \operatorname{Li}_{2}(a)-\frac{1}{2} \ln (1-a)+O\left(\ln \frac{1}{q}\right) .
\end{aligned}
$$

The second factor in the correlation function (4.11) can be obtained by the inversion $a \rightarrow 1 / a$. Thus, combining them together and making use of the obvious relations,

$$
\begin{aligned}
& \mathrm{Li}_{2}\left(\mathrm{e}^{2 \pi i \alpha}\right)+\mathrm{Li}_{2}\left(\mathrm{e}^{-2 \pi i \alpha}\right)=2 \pi^{2}\left(\frac{1}{6}-\alpha(1-\alpha)\right), \\
& \ln \left(1-\mathrm{e}^{2 \pi i \alpha}\right)+\ln \left(1-\mathrm{e}^{-2 \pi i \alpha}\right)=2 \ln (2 \sin (\pi \alpha)),
\end{aligned}
$$

one can find the scaling dimension and the normalization constant in Eq. (4.28). The $a=1$ case has to be studied separately, as one can see the appearance of divergences in the expansion (A3). In this regime, we merely quote the result by Kluyver [40],

$\ln \prod_{k=1}^{\infty}\left(1-q^{k}\right)=-\frac{\zeta(2)}{\ln \frac{1}{q}}-\frac{1}{2} \ln \ln \frac{1}{q}+\frac{1}{2} \ln (2 \pi)+\frac{1}{24} \ln \frac{1}{q}+\cdots$,

which was a predecessor to the Watson estimation [41], i.e., Eq. (4.17).
[1] L. J. Dixon, D. Friedan, E. J. Martinec, and S. H. Shenker, The conformal field theory of orbifolds, Nucl. Phys. B282, 13 (1987).

[2] V. G. Knizhnik, Analytic fields on Riemann surfaces. 2, Commun. Math. Phys. 112, 567 (1987).

[3] M. Bershadsky and A. Radul, g-loop amplitudes in bosonic string theory in terms of branch points, Phys. Lett. B 193, 213 (1987).

[4] C. Holzhey, F. Larsen, and F. Wilczek, Geometric and renormalized entropy in conformal field theory, Nucl. Phys. B424, 443 (1994).

[5] P. Calabrese and J. L. Cardy, Entanglement entropy and quantum field theory, J. Stat. Mech. (2004) P06002.

[6] J. L. Cardy, O. A. Castro-Alvaredo, and B. Doyon, Form factors of branch point twist fields in quantum integrable models and entanglement entropy, J. Stat. Phys. 130, 129 (2007).

[7] B. Basso, A. Sever, and P. Vieira, Space-Time and Flux Tube S-Matrices at Finite Coupling for $N=4$ Supersymmetric Yang-Mills Theory, Phys. Rev. Lett. 111, 091602 (2013).

[8] B. Basso, A. Sever, and P. Vieira, Space-time S-matrix and flux tube S-matrix II. Extracting and matching data, J. High Energy Phys. 01 (2014) 008.
[9] A. V. Belitsky, Nonsinglet pentagons and NMHV amplitudes, Nucl. Phys. B896, 493 (2015).

[10] L. F. Alday and J. M. Maldacena, Gluon scattering amplitudes at strong coupling, J. High Energy Phys. 06 (2007) 064.

[11] J. M. Drummond, J. Henn, G. P. Korchemsky, and E. Sokatchev, On planar gluon amplitudes/Wilson loops duality, Nucl. Phys. B795, 52 (2008).

[12] A. Brandhuber, P. Heslop, and G. Travaglini, MHV amplitudes in $N=4$ super Yang-Mills and Wilson loops, Nucl. Phys. B794, 231 (2008).

[13] L. F. Alday and J. M. Maldacena, Comments on operators with large spin, J. High Energy Phys. 11 (2007) 019.

[14] L. F. Alday, D. Gaiotto, and J. Maldacena, Thermodynamic bubble ansatz, J. High Energy Phys. 09 (2011) 032.

[15] B. Basso, A. Sever, and P. Vieira, Collinear Limit of Scattering Amplitudes at Strong Coupling, Phys. Rev. Lett. 113, 261604 (2014).

[16] B. Schroer and T. T. Truong, The order/disorder quantum field operators associated to the two-dimensional Ising model in the continuum limit, Nucl. Phys. B144, 80 (1978).

[17] A. V. Belitsky, Nonperturbative enhancement of superloop at strong coupling, Nucl. Phys. B911, 425 (2016); Twisting perturbed parafermions, Phys. Lett. B 770, 35 (2017). 
[18] A. Bonini, D. Fioravanti, S. Piscaglia, and M. Rossi, The contribution of scalars to $N=4$ SYM amplitudes, Phys. Rev. D 95, 041902 (2017).

[19] V. Gurarie, Logarithmic operators in conformal field theory, Nucl. Phys. B410, 535 (1993).

[20] M. Sato, T. Miwa, and M. Jimbo, Holonomic quantum fields. 4., Publ. Res. Inst. Math. Sci. Kyoto 15, 871 (1979).

[21] L. P. Kadanof and H. Ceva, Determination of an operator algebra for the two-dimensional Ising model, Phys. Rev. B 3, 3918 (1971).

[22] E. C. Marino, B. Schroer, and J. A. Swieca, Euclidean functional integral approach for disorder variables and kinks, Nucl. Phys. B200, 473 (1982).

[23] B. Schroer, Functional integrals for order-disorder variables in terms of Aharonov-Bohm strings, Nucl. Phys. B210, 103 (1982).

[24] A. Kokotov, Compact polyhedral surfaces of an arbitrary genus and determinants of Laplacians, arXiv:0906.0717.

[25] L. Hillairet and A. Kokotov, Krein formula and S-matrix for Euclidean surfaces with conical singularities, J. Geomet. Anal. 23, 1498 (2013); L. Hillairet, V. Kalvin, and A. Kokotov, Moduli spaces of meromorphic functions and determinant of Laplacian, arXiv:1410.3106.

[26] P. Loya, P. Mcdonald, and J. Park, Zeta regularized determinants for conic manifolds, J. Funct. Anal. 242, 195 (2007).

[27] D. B. Ray and I. M. Singer, R-torsion and the Laplacian on Riemannian manifold, Adv. Math. 7, 145 (1971).

[28] S. W. Hawking, Zeta function regularization of path integrals in curved space-time, Commun. Math. Phys. 55, 133 (1977).

[29] A. V. Belitsky, Vacuum expectation value of twist fields, Phys. Rev. D 96, 066026 (2017).

[30] I. M. Gelfand and A. M. Yaglom, Integration in functional spaces and it applications in quantum physics, J. Math. Phys. (N.Y.) 1, 48 (1960).

[31] G. V. Dunne, Functional determinants in quantum field theory, J. Phys. A 41, 304006 (2008).

[32] G. N. Watson, A Treatise on the Theory of Bessel Functions (Cambridge University Press, Cambridge, 1922).

[33] J. Brüning and R. Seeley, Regular singular asymptotics, Adv. Math. 58, 133 (1985); The resolvent expansion for second order regular singular operators, J. Funct. Anal. 73, 369 (1987).

[34] M. Spreafico, On the nonhomogeneous quadratic Bessel zeta function, Mathematika 51, 123 (2004); Zeta function and regularized determinant on a disc and on a cone, J. Geom. Phys. 54, 355 (2005).

[35] F. W. J. Olver, Asymptotics and Special Functions (Academic Press, New York, 1974).

[36] A. M. Polyakov, Quantum geometry of bosonic strings, Phys. Lett. 103B, 207 (1981).

[37] O. Alvarez, Theory of strings with boundaries: Fluctuations, topology, and quantum geometry, Nucl. Phys. B216, 125 (1983).

[38] B. Osgood, R. Phillips, and P. Sarnak, Extremals of determinants of Laplacians, J. Funct. Anal. 80, 148 (1988).

[39] G. E. Andrews, q-Series: Their Development and Application in Analysis, Number Theory, Combinatorics, Physics, and Computer Algebra (American Mathematical Society, Providence, 1986).
[40] J. C. Kluyver, On Lambert's series, KNAW Proc. 22 I, 323 (1919).

[41] G. N. Watson, The final problem: An account of the Mock theta functions, J. Lond. Math. Soc. s1-11, 55 (1936).

[42] S. Banerjee and B. Wilkerson, Asymptotic expansions of Lambert series and related q-series, Int. J. Number Theory 13, 2097 (2017); I. Mezo, On asymptotic estimation of the $q$-Pochhammer symbols at $q=1$, Int. J. App. Math. 26, 511 (2013).

[43] D. Bianchini and O. A. Castro-Alvaredo, Branch point twist field correlators in the massive free boson theory, Nucl. Phys. B913, 879 (2016).

[44] O. Blondeau-Fournier and B. Doyon, Expectation values of twist fields and universal entanglement saturation of the free massive boson, J. Phys. A 50, 274001 (2017).

[45] A. Weil, Elliptic Functions According to Eisenstein and Kronecker (Springer-Verlag, New York, 1976).

[46] P. Epstein, Zur Theorie allgemeiner Zetafunctionen, Math. Ann. 56, 615 (1903); Zur Theorie allgemeiner Zetafunktionen, II, Math. Ann. 63, 205 (1906).

[47] S. Lukuyanov and A. B. Zamolodchikov, Exact expectation values of local fields in quantum sine-Gordon model, Nucl. Phys. B493, 571 (1997).

[48] M. F. Atiyah, V. K. Patodi, and I. M. Singer, Spectral asymmetry and Riemannian geometry, I., Math. Proc. Cambridge Philos. Soc. 77, 43 (1975); Spectral asymmetry and Riemannian geometry, II., Math. Proc. Cambridge Philos. Soc. 78, 405 (1975); Spectral asymmetry and Riemannian geometry, III., Math. Proc. Cambridge Philos. Soc. 79, 71 (1976).

[49] M. Hortacsu, K. D. Rothe, and B. Schroer, Zero-energy eigenstates for the Dirac boundary problem, Nucl. Phys. B171, 530 (1980).

[50] B. M. McCoy, C. A. Tracy, and T. T. Wu, Painleve functions of the third kind, J. Math. Phys. (N.Y.) 18, 1058 (1977).

[51] H. Casini and M. Huerta, Entanglement and alpha entropies for a massive scalar field in two dimensions, J. Stat. Mech. (2005) P12012.

[52] W. Müller, Relative determinants of elliptic operators and scattering theory, J. Equ. Deriv. Partielles 13, 24 (1996).

[53] I. M. Lifshitz, On a problem in perturbation theory connected with quantum statistics, Usp. Mat. Nauk 7, 171 (1952) (in Russian).

[54] M. G. Krein, On a trace formula in perturbation theory, Mat. Sb. 33, 597 (1953) (in Russian).

[55] M. S. Birman and M. G. Krein, On the theory of wave operators and scattering operators, Sov. Math. Dokl. 144, 745 (1962) (in Russian).

[56] M. S. Birman and D. R. Yafaev, The spectral shift function. The papers of M. G. Krein and their further development, Algebra and Analysis 4, 1 (1992) (in Russian).

[57] E. Bogomolny, S. Mashkevich, and S. Ouvry, Scattering on two Aharonov-Bohm vortices with opposite fluxes, J. Phys. A 43, 354029 (2010); E. Bogomolny, Scattering on two Aharonov-Bohm vortices, J. Phys. A 49, 485202 (2016).

[58] D. Burghelea, L. Friedlander, and T. Kappeler, MeyerVietoris type formula for determinants of elliptic differential operators, J. Funct. Anal. 107, 34 (1992). 